\title{
A note on reference solution based $h p$-adaptive PDE solvers
}

\author{
Tamás L. Horváth
}




\title{
A NOTE ON REFERENCE SOLUTION BASED $H P$-ADAPTIVE PDE SOLVERS
}

\author{
TAMÁS L. HORVÁTH
}

Received 24 September, 2013

\begin{abstract}
In this note we suggest an improvement for a family of $h p$-adaptive finite element methods. We point out the necessity of the new procedure by constructing model problems for which certain standard $h p$-adaptive algorithms fail to work properly. It is verified also in the corresponding simulations that the algorithm can terminate even though the numerical solution still contains a sizable computational error.
\end{abstract}

2010 Mathematics Subject Classification: 65 N30

Keywords: reference solution, counterexample, $h p$-adaptivity

\section{INTRODUCTION}

There are several $h p$-adaptive finite element algorithms in the literature for the numerical solution of PDE's (see the collection [8] and the references therein). These are based on the following scheme

Initialize: solve the initial problem with small polynomial degree $p$ on a coarse grid,

\section{Repeat:}

S1 estimate the error,

$\mathrm{S} 2$ if the error is small then stop,

S3 else determine on which elements in the grid and how to refine/derefine,

$\mathrm{S} 4$ compute the new solution and go to $\mathrm{S} 1$.

The main differences between the different methods are in the error estimation and refinement procedures. Here we will focus on the method introduced by Demkowicz et al. [2] and also used (with a small modification) by Šolín et al. [11]. In step S1 this method uses the so called "reference solution" to compute the error. This reference solution is calculated using a uniform refinement in space and by increasing the degree of polynomials on every element in order to calculate a finer solution that will be considered as the reference solution. The error is defined as the difference between the reference solution and original solution. 
Despite the fact that this method can be used for many different problems, such as time dependent problems [4,13], Maxwell's equations [12,14] or coupled highly nonlinear problems [5], the efficiency of the error estimation has not been justified rigorously. We illustrate this by the so-called antenna example [3, Section 15.3.] where the convergence of the method seems to be broken because the estimated error does not decrease during refinements. However, after further iteration steps it decreases again until the estimated error becomes small enough. In that case the error estimator works properly because it forces the refinements until the estimated error is small enough.

The aim of this paper is to give a counterexample where the reference solution is the same as the original one, hence the computed error will be zero, whereas the error between the exact solution and the approximate solution is large. Furthermore we suggest a modification of the method that can be used to avoid these kinds of difficulties.

\section{NOTATIONS}

We investigate the elliptic boundary-value problem

$$
\begin{aligned}
-\operatorname{div}(K \nabla u)+C u & =f & & \text { in } \Omega, \\
u & =g & & \text { on } \Gamma,
\end{aligned}
$$

and its weak form. Find $u \in H^{1}(\Omega)$ such that $u=u_{D}+\phi$ where $u_{D}=g$ on $\partial \Omega$, $\phi \in H_{0}^{1}(\Omega)$

$$
\begin{aligned}
& a(\phi, v)=\int_{\Omega} f v-a\left(u_{D}, v\right) \quad \forall v \in H_{0}^{1}(\Omega), \\
& a(\phi, v):=\int_{\Omega} K \nabla \phi \cdot \nabla v+\int_{\Omega} C \phi v,
\end{aligned}
$$

where $\Omega \subset \mathbb{R}^{d}, d \geq 1, \Gamma=\partial \Omega, f \in L_{2}(\Omega), C \in L_{\infty}(\Omega), K=\left\{k_{i, j}\right\}_{i, j=1}^{d}$ is a continuous, matrix valued, symmetric function on $\bar{\Omega}$ and $v \in H_{0}^{1}(\Omega)$ is a test function.

Let us denote by $\tau_{h}$ a tessallation of $\Omega$ into elements. The discretization of (2.3) takes the following form. Let us denote by $V_{h, p} \subset H^{1}(\Omega)$ a finite dimensional subspace. Find $u_{h, p} \in V_{h, p}$ such that $u_{h, p}=u_{D_{h, p}}+\phi_{h, p}$ where $u_{D_{h, p}} \in V_{h, p}$ approximates $g$ on $\partial \Omega, \phi \in V_{h, p}$

$$
a\left(\phi_{h, p}, v_{h, p}\right)=\int_{\Omega} f v_{h, p}-a\left(u_{D_{h, p}}, v_{h, p}\right) \quad \forall v \in V_{h, p} .
$$

$V_{h, p}$ contains piecewise polynomials whose degrees can vary from element to element.

$V_{h / 2, p+1}$ denotes the approximation space where the mesh is refined and the degree of the local polynomials is increased. 
The reference solution based methods described in [2] and [11] use the following basic idea:

S1 compute $u_{h, p} \in V_{h, p}$ by solving (2.4),

S2 compute the reference solution $u_{h / 2, p+1} \in V_{h / 2, p+1}$ by solving (2.4) in the enriched space $V_{h / 2, p+1}$,

S3 use $u_{h / 2, p+1}$ as a more accurate solution and define $u_{h, p}-u_{h / 2, p+1}$ as an error indicator,

S4 compute one of the following quantities on all $T \in \tau_{h}$

- $\eta_{T}=\frac{\left|u_{h, p}-u_{h / 2, p+1}\right|_{H^{1}(T)}}{\left|u_{h / 2, p+1}\right|_{H^{1}(T)}}$

- $\eta_{T}=\frac{\left\|u_{h, p}-u_{h / 2, p+1}\right\|_{H^{1}(T)}}{\left\|u_{h / 2, p+1}\right\|_{H^{1}(T)}}$

- $\eta_{T}=\left|u_{h, p}-u_{h / 2, p+1}\right|_{H^{1}(T)}$

- $\eta_{T}=\left\|u_{h, p}-u_{h / 2, p+1}\right\|_{H^{1}(T)}$

S5 if $\sqrt{\sum_{T} \eta_{T}^{2}}<$ TOL stop, else refine where it is needed, and go to S1.

The difference between [2] and [11] lies in the choice of $\eta_{T}$ and [2] does a refinement all over the edges not only all over the elements.

\section{Counterexample}

The construction of the counterexample is based on the following simple idea. Let us suppose that we can find a function $f \neq 0$ such that $\int_{\Omega} f v_{h, p}=0, \forall v_{h, p} \in V_{h, p}$. In this case (2.4) simplifies to $a\left(\phi_{h, p}, v_{h, p}\right)=a\left(u_{D_{h, p}}, v_{h, p}\right)$. Suppose that it has an exact solution $u_{0} \in V_{h, p}$ so $u_{h, p}=u_{0}$ (i.e. if $g=0$ then $u_{D}=0$ yielding $u_{0}=0$ ).

If $\int_{\Omega} f v_{h / 2, p+1}=0, \forall v_{h / 2, p+1} \in V_{h / 2, p+1}$ then using the fact $V_{h, p} \subset V_{h / 2, p+1}$ we have that $\int_{\Omega} f v_{h, p}=0$ also holds. In this case $u_{h, p}=u_{h / 2, p+1}=u_{0}$, and therefore the computed error is zero.

Now we show how to create a function $f$ satisfying the above assumptions. For any $T \in \tau_{h}$ we define $u_{c}: \Omega \rightarrow \mathbb{R}$ such that $\operatorname{supp}\left(u_{c}\right)=T, u_{c}(x, y)=$ $=\chi_{T}(x, y) b_{T}^{2}(x, y) p(x, y)$, where $\chi_{T}$ is the characteristic function of $T, b_{T}: \Omega \rightarrow$ $\mathbb{R}$ is a bubble function on $T$, so $b_{T}=0$ on $\partial T$ and

$$
p(x, y)=\sum_{k=0}^{m-1} c_{k} x^{a_{k}} y^{b_{k}} .
$$

Here $a_{k}, b_{k} \in \mathbb{N} \cup\{0\}$ and $c_{k} \in \mathbb{R}, m$ is a fixed integer (that will be determined later). The polynomial $p(x, y)$ is chosen so that

$$
\int_{\Omega}\left(-\operatorname{div}\left(K \nabla u_{c}\right)+C u_{c}\right) v=0 \quad \forall v: v \in V_{h / 2, p+1}, \operatorname{supp}(v)=T,
$$


or, since $\operatorname{supp}\left(u_{c}\right)=T$

$$
\int_{T}\left(-\operatorname{div}\left(K \nabla u_{c}\right)+C u_{c}\right) v=0 \quad \forall v: v \in V_{h / 2, p+1} .
$$

In order to compute the coefficient vector $c=\left(c_{0}, \ldots, c_{m-1}\right)$ we have to solve the linear system $A c=0$ where the entries of $A \in \mathbb{R}^{n \times m}$ are given by

$$
A_{i, j}=\int_{T}\left(-\operatorname{div}\left(K \nabla b_{T}^{2} c_{j} x^{a_{j}} y^{b_{j}}\right)+C b_{T}^{2} c_{j} x^{a_{j}} y^{b_{j}}\right) v_{i} d T \quad \forall i \in\{1,2, \ldots, n\} .
$$

Here we have used the notation $\left.\operatorname{dim} V_{h / 2, p+1}\right|_{T}=n$. To find a nonzero solution $c, A$ must have more columns than rows, so $m:=n+1$. It is sufficient to find a submatrix $\bar{A} \in \mathbb{R}^{r(A) \times r(A)+1}$ where $r(A)$ is the rank of $A$, and solve the reduced system $\bar{A} \bar{c}=0$.

If we have such a solution then we have at least one free parameter to define $c$. We set this parameter to an arbitrary number, i.e. 1 . This can be used as coefficients $c$ in the definition of $u_{c}$.

For any $C_{0} \in \mathbb{R}$ the test function $u_{0}+C_{0} u_{c}$ will give $u_{h, p}=u_{h / 2, p+1}=u_{0}$ and the algorithm will terminate, even though the error $C_{0} u_{c}$ can be arbitrarily large.

Remark 1. If we use implicit a posteriori error estimation first we solve (2.4) which gives $u_{h, p}=u_{0}$. Then a local Neumann problem is solved on every element $\widetilde{T}$

$$
\begin{aligned}
-\operatorname{div}(K \nabla e)+C e & =f+\operatorname{div}\left(K \nabla u_{h, p}\right)-C u_{h, p} \quad \text { in } \widetilde{T}, \\
\frac{\partial e}{\partial \eta} & =-\frac{1}{2}\left[\frac{\partial u_{h p}}{\partial \eta}\right] \quad \text { on } \partial \widetilde{T} \backslash \partial \Omega, \\
e & =0 \quad \text { on } \partial \widetilde{T} \cap \partial \Omega,
\end{aligned}
$$

where $e$ is an estimator of $u-u_{h, p},\left[\frac{\partial u_{h p}}{\partial \eta}\right]$ is the jump of the outward normal derivative of the numerical solution on an interior edge (see Chapter 3 in [1] for details). If $u_{0}=0$ on $\mathrm{T}$ then the r.h.s. of (3.4) will be $f$. The estimated boundary condition will also be zero. The solution of (3.4)-(3.6) depends on the local finite element space $W_{\widetilde{T}}$. If $W_{\widetilde{T}} \subseteq V_{h / 2, p+1}$, then we will again have $e=0$ on $\widetilde{T}$.

\section{NUMERICAL RESULTS}

By courtesy of William F. Mitchell the procedure described above was tested numerically using his PHAML code [7]. The code was supplied with the following initial mesh:

The problem was a simple Poisson equation, $K \equiv 1, C \equiv 0$, with Dirichlet boundary condition in (2.1)-(2.2)

$$
\begin{aligned}
-\triangle u(x, y) & =f(x, y) \quad \text { in } \Omega, \\
u(x, y) & =u_{D}(x, y) \quad \text { on } \Gamma,
\end{aligned}
$$






FIGURE 1. The initial mesh and triangle $T$.

with $\Omega=(0,1)^{2}$, and $b_{k}=0$ (see (3.1)). The polynomial degree $p$ was 1 at the initial step. For computing the reference solution PHAML used bisected triangles.

We used as our counterexample

$$
\begin{aligned}
u_{c}(x, y)= & x+C_{0} \chi_{T}(x, y)\left(-3200(x-y)^{2} y^{2}(2 x-1)^{2}(-1+4 x)^{2} .\right. \\
& \left(1810432 x^{7}-4313088 x^{6}+4323072 x^{5}-2356224 x^{4}\right. \\
& \left.\left.+751088 x^{3}-139176 x^{2}+13747 x-549\right)\right),
\end{aligned}
$$

and $f_{c}(x, y):=-\Delta u_{c}(x, y), u_{D}(x, y)=\left.x\right|_{\Gamma}, C_{0}=10^{5}$.

The second term of $u_{c}$ was calculated by the method described above. Theoretically we should have $u_{h, p}=u_{h / 2, p+1}=x$ according to the previous section, yielding that the real error $\left\|u-u_{h, p}\right\|_{H^{1}}$ can be arbitrary and we can control it by our choice of $C_{0}$.

When we implemented this model problem in PHAML we encountered problems with numerical integration. One can verify that $\int_{T_{j}} f_{c}(x, y) \cdot x^{k} y^{l}=0$ if $0 \leq k, l \leq 2$, $k+l \leq 2$ for all $T_{j}$ that are a subtriangle of $T$ and for any $C_{0}$. However, even when the highest available order quadrature was used it was different from zero and the r.h.s. of (2.3) became nonzero.

We obtained $\left\|u_{h / 2, p+1}-u_{h, p}\right\|_{H^{1}(\Omega)} \approx 10^{-9}$, which was our main aim. The addition of $x$ was necessary. Without it $\left\|u_{h / 2, p+1}\right\|_{H^{1}(\Omega)} \approx 10^{-9}$ and the relative error was $O(1)$. The addition of 1 would not make any difference if we used a seminorm instead of a norm. 
For all possible stopping criteria mentioned in Section 2 we could achieve $\sqrt{\sum_{T} \eta_{T}^{2}}$ $\approx 10^{-9}$. This means that the algorithm terminated at the initial step whenever TOL $>$ $10^{-8}$, even though the computational error can be almost arbitrary, depending only on $C_{0}$.

\section{CONCLUSION}

We can easily fix this problem by building a back-up estimator into the code. For example, we can use the residual-based error estimator

$$
\left\|u-u_{h, p}\right\|^{2} \leq \eta_{\mathrm{res}}^{2}=C_{\mathrm{res}}\left(\sum_{T \in \tau_{h}} h_{T}^{2}\|r\|_{L_{2}(T)}^{2}+\sum_{\gamma \in \partial T} h_{T}\|R\|_{L_{2}(\gamma)}^{2}\right),
$$

where $r$ is the interior residual $r=f+\operatorname{div}\left(K \nabla u_{h, p}\right)-C u_{h, p}, R=\left[\frac{\partial u_{h p}}{\partial \eta}\right]$ is the jump of the derivative of the numerical solution on the interior edges, $\|u\| \|^{2}=a(u, u)$ is the energy norm (see [1] for details), and $C_{\text {res }}$ is a constant which does not depend on $h$.

It is well known that reference solution based methods are a very effective class of adaptive techniques. The inequality (5.1) supplies a guaranteed upper bound; on the other hand, its use for the purpose of $h p$-adaptivity is a little bit complicated. We should modify our algorithm as follows:

Initialize: solve the initial problem with small polynomial degree $p$ on a coarse grid

\section{Repeat:}

S1 compute the error using one of the quantities from Step 4 of the algorithm described at the end of Section 2.

$\mathrm{S} 2$ if the error is small then use (5.1)

S2a if $\eta_{\text {res }}<$ TOL terminate

S2b else do a brute-force adaptive step (both $h$ and $p$ ) and go to step S4. (See Remark 3.)

S3 else determine on which elements in the grid and how to refine/derefine

$\mathrm{S} 4$ compute the new solution and go to $\mathrm{S} 1$

Remark 2. By adopting this strategy we can avoid the need for building an effective $h p$-adaptive technique that impinges on a residual-based estimator. We propose to use a brute-force adaptive step in order to try to avoid a major modification of the original algorithm. It is possible to use the $h p$-adaptive technique from [6] that provides lower degrees of freedom, however, the method would become more complicated.

Remark 3. There exists another possible correction that was published in $[9,10]$. Those papers introduce an adaptive method that is proved to be convergent. The key idea is the measurement of the oscillation of $f$. Elements should be checked twice: 
first according to the error estimator and after that according to the oscillation of the right hand side. If the refinement involves all problematic elements the method will converge. In our case the only question would be how to decide which refining option should be used over the elements where there is considerable oscillation: they could be refined both in $h$ and $p$.

The reference solutions are characterized by one fundamental issue: they assume a local behavior of the error. This means that in most cases the error can be located easily if it is caused by a rough mesh or low polynomial degree at the place where it is detected. Otherwise, when the error is spread around the reference solution methods fail. However, above we have seen that it can also fail to converge to the exact solution even when the error is caused by low polynomial degree on an element.

The idea of creating the counterexample, can be used for many applications. Although, we should note that the way of the correction is a more difficult question. The residual-based error estimator is hard to carry out for every type of PDEs, especially with the proper constant. On the other hand, the reference solution based algorithm is easy-to-use for wide range of problems.

\section{ACKNOWLEDGEMENT}

The author is grateful to William F. Mitchell for his help on PHALM, and to Endre Süli for his encouragement to writing this note.

The author acknowledges support from OTKA (grant no. K81403).

\section{REFERENCES}

[1] M. Ainsworth and J. T. Oden, A posteriori error estimation in finite element analysis, ser. Pure and Applied Mathematics (New York). New York: Wiley-Interscience [John Wiley \& Sons], 2000.

[2] L. Demkowicz, W. Rachowicz, and P. Devloo, "A fully automatic $h p$-adaptivity," in Proceedings of the Fifth International Conference on Spectral and High Order Methods (ICOSAHOM-01) (Uppsala), vol. 17, no. 1-4, 2002, pp. 117-142.

[3] L. Demkowicz, Computing with hp-adaptive finite elements. Vol. 1, ser. Chapman \& Hall/CRC Applied Mathematics and Nonlinear Science Series. Chapman \& Hall/CRC, Boca Raton, FL, 2007, one and two dimensional elliptic and Maxwell problems, With 1 CD-ROM (UNIX).

[4] L. Dubcová, P. Šolín, J. Červený, and P. Kus, "Space and time adaptive two-mesh hp-fem for transient microwave heating problems," Electromagnetics, vol. 30, no. 1-2, pp. 23-40, 2009.

[5] L. Dubcová, P. Šolín, G. Hansen, and H. Park, "Comparison of multimesh $h p$-fem to interpolation and projection methods for spatial coupling of thermal and neutron diffusion calculations," $J$. Comput. Phys., vol. 77, pp. 1182-1197, 2011.

[6] P. Houston, B. Senior, and E. Süli, "Sobolev regularity estimation for $h p$-adaptive finite element methods," in Numerical mathematics and advanced applications. Springer Italia, Milan, 2003, pp. 631-656.

[7] W. F. Mitchell, PHAML User's Guide. NIST, 2006.

[8] W. F. Mitchell and M. A. McClain, "A survey of $h p$-adaptive strategies for elliptic partial differential equations," Recent Advances in Computational and Applied Mathematics, pp. 227-258, 2011. 
[9] P. Morin, R. H. Nochetto, and K. G. Siebert, "Data oscillation and convergence of adaptive FEM," SIAM J. Numer. Anal., vol. 38, no. 2, pp. 466-488 (electronic), 2000. [Online]. Available: http://dx.doi.org/10.1137/S0036142999360044

[10] P. Morin, R. H. Nochetto, and K. G. Siebert, "Convergence of adaptive finite element methods," SIAM Rev., vol. 44, no. 4, pp. 631-658 (electronic) (2003), 2002, revised reprint of "Data oscillation and convergence of adaptive FEM" [SIAM J. Numer. Anal. 38 (2000), no. 2, 466-488 (electronic); MR1770058 (2001g:65157)]. [Online]. Available: http://dx.doi.org/10.1137/S0036144502409093

[11] P. Šolín, J. Červený, and I. Doležel, "Arbitrary-level hanging nodes and automatic adaptivity in the hp-fem," Math. Comput. Simulation, vol. 77, pp. 117-132, 2008.

[12] P. Šolín, L. Dubcová, J. Červený, and I. Doležel, "Adaptive hp-fem with arbitrary-level hanging nodes for maxwell's equations," Math. Comput. Simul., vol. 17, pp. 127-155, 2009.

[13] P. Šolín, K. Segeth, and I. Doležel, "Space-time adaptive $h p$-FEM: methodology overview," in Programs and algorithms of numerical mathematics 14. Acad. Sci. Czech Repub. Inst. Math., Prague, 2008, pp. 185-200.

[14] P. Šolín, L. Dubcová, and Doležel, Ivo, "Adaptive $h p$-FEM with arbitrary-level hanging nodes for Maxwell's equations,” Adv. Appl. Math. Mech., vol. 2, no. 4, pp. 518-532, 2010.

Author's address

Tamás L. Horváth

Department of Applied Analysis and Computational Mathematics, Eötvös Loránd University, H1117 Budapest, Pázmány P. sétány 1/C, Hungary

MTA-ELTE Numerical Analysis and Large Networks Research Group, H-1117 Budapest, Pázmány P. sétány $1 / C$, Hungary

E-mail address: thorvathecs.elte.hu 